\title{
Rare skin and testicular extranodal involvement in a patient with marginal zone lymphoma on 18F-FDG PET-CT imaging
}

\author{
Pelin Ozcan Kara ${ }^{*}$, Zehra Pinar Koc ${ }^{1}$, Emel Sezer ${ }^{2}$ and Kadir Eser ${ }^{2}$ \\ ${ }^{1}$ Mersin University, Faculty of Medicine, Department of Nuclear Medicine, Mersin, Turkey \\ ${ }^{2}$ Mersin University, Faculty of Medicine, Department of Oncology, Mersin, Turkey
}

\begin{abstract}
Hodgkin lymphoma (HL) and Non-Hodgkin lymphoma (NHL) are the malignant neoplasm spectrum originating from the lymphoid system and constituting 8\% of all malignancies. Although, both are known as primary malignancies of lymph nodes, extranodal involvement can be seen. In this case presentation, in addition to nodal and bone involvement areas in a patient diagnosed as marginal zone lymphoma, rare simultaneous testicular and skin extranodal involvement detected on 18F-FDG PET-CT imaging were presented.
\end{abstract}

Abbreviations: Hodgkin lymphoma-HL, Non-Hodgkin lymphomaNHL, Marginal zone lymphomas-MZL

\section{Introduction}

Extranodal involvement areas such as gastrointestinal, head and neck, orbital, central and peripheral nervous system, thorax, bone, skin, breast, testis, thyroid and genitourinary system can be seen in $25-40 \%$ of HL and especially NHL patients, although it is known as lymph node malignancy [1,2]. Extranodal involvement is important in terms of prognosis and imaging procedures play a very important role in diagnosis. 18F-FDG PET-CT imaging is a standard method in lymphoma patients and has a special importance in these patients in terms of superiority of CT imaging in detecting extranodal regions. The role of 18F-FDG PET-CT in detection, staging and restaging of patients with extranodal involvement in NHL has also been reported in the literature $[3,4]$. In this case presentation, in addition to nodal and bone involvement areas in a patient diagnosed as marginal zone lymphoma, rare testicular and skin extranodal simultaneous involvement detected on 18F-FDG PET-CT imaging were presented.

\section{Case report}

A 72-year-old male patient diagnosed as marginal zone lymphoma following mass excision from the left thoracic cage underwent 18F-FDG PET-CT imaging for initial staging. PET-CT images showed hypermetabolic (SUVmax: 14.66-31.89) conglomereted lymphadenopathies in the bilateral cervical chain, supraclavicular area, bilateral axillary, mediastinal, retroperitoneal, paraaortic, peripancreatic, perigastric, liver hilum, paracoeliac regions, pericardial thickening-effusion and hypermetabolic (SUVmax: 3.32-12.83) bone lesions. Additionally, multiple subcutaneous hypermetabolic (SUVmax: 10.5-24.37) nodules were detected at various levels within the cross-sectional area. In addition; Testis lymphoma FDG uptake (SUVmax: 20.26) was noted in the right hemiscrotum (Figure 1). Testis lymphoma has been proved by excisional biopsy.

\section{Discussion}

Marginal zone lymphomas (MZL) are defined as a heterogeneous group of lymphomas although, can originate from the spleen, lymph nodes and extra-nodal lymphoid tissue, termed as "marginal zone" and formed of B-lymphocytes. Testicular involvement is a very rare entity. Testicular lymphoma can be primary or secondary to extensive

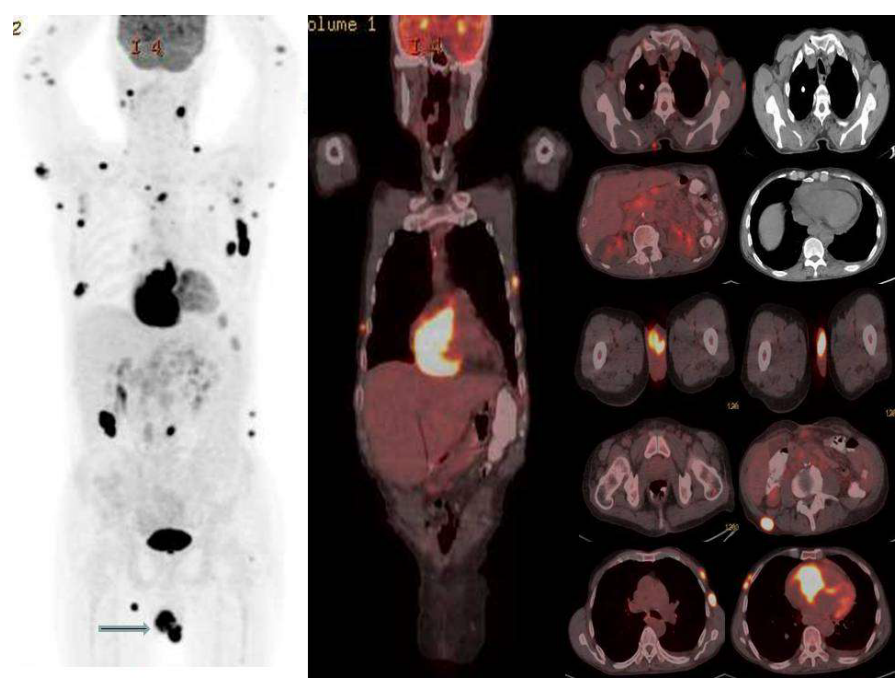

Figure 1. 18F-FDG PET-CT Image showing the spread of Lymphoma.

Correspondence to: Pelin Ozcan Kara, 1Mersin University, Faculty of Medicine, Department of Nuclear Medicine, Mersin, Turkey, Tel: +90-324-241-5000; E-mail: ppelinozcan@gmail.com

Key words: rare skin, testicular extranodal, PET-CT imaging, marginal zone lymphoma, nuclear medicine, Hodgkin lymphoma, Non-Hodgkin lymphoma

Received: May 28, 2017; Accepted: June 26, 2017; Published: June 29, 2017 
disease. Inguinal and retroperitoneal lymph nodal spread can also be seen in some patients [3]. In our case, there was abnormally increased asymmetric FDG uptake in the enlarged right testis. Also, multiple para-aortic lymph nodes showed abnormal FDG accumulation, as if spreading from involved testis by lymphomatous way. Yin et al. reported a case of a 55-year-old man with NHL of the left testis and of the bilateral adrenals detected by 18 F-FDG PET-CT and demonstrated its role in the diagnosis and assessment of therapeutic response [5]. Multiple isolated cases with extranodal involvement of NHL, detected on PET-CT, have been previously reported [5-8]. In this case report, in addition to nodal involvement areas in a patient diagnosed as marginal zone lymphoma, rare areas of testicular and skin extranodal involvement detected by $18 \mathrm{~F}-\mathrm{FDG}$ PET-CT imaging is presented. Patients with abnormal testicular involvement should undergo further examination and confirmation of the finding is necessary.

\section{References}

1. Lopez-Guillermo A, Colomo L, Jimenez M, Bosch F, Villamor N, et al. (2005) Diffuse large B-cell lymphoma: clinical and biological characterization and outcome according to the nodal or extranodal primary origin. J Clin Oncol 23: 2797-804. [Crossref]
2. Economopoulos T, Papageorgiou S, Rontogianni D, Kaloutsi V, Fountzilas G, et al. (2005) Multifocal Extranodal Non-Hodgkin Lymphoma: a clinicopathologic study of 37 Cases in Greece, a Hellenic Cooperative Oncology Group Study. Oncologist 10: 734-8. [Crossref]

3. Paes FM, Kalkanis DG, Sideras PA, Serafini AN. (2010) FDG PET/CT of Extranodal Involvement in Non-Hodgkin Lymphoma and Hodgkin Disease. RadioGraphics 30: 269-291.

4. Even-Sapir E, Lievshitz G, Perry C, Herishanu Y, Lerman H, et al. (2007) Fluorine-18 Fluorodeoxyglucose PET/CT Patterns of Extranodal Involvement in Patients with NonHodgkin Lymphoma and Hodgkin's Disease. Radiol Clin N Am 45: 697-709. [Crossref]

5. Yin Y, Qing F, Li X, Du B, Li N, et al. (2011) Non-Hodgkin's lymphoma of the testicle and bilateral adrenals detected by 18F-FDG PET/CT. Exp Theor Med 2: 817-820. [Crossref]

6. Julian A, Wagner T, Ysebaert L, Chabbert V, Payoux P. (2011) FDG PET/CT leads to the detection of metastatic involvement of the heart in non-Hodgkin'slymphoma. Eur J Nucl Med Mol Imaging 38: 1174.

7. Kaderli AA, Baran I, Aydin O, Bicer M, Akpinar T, et al. (2010) Diffuse involvement of the heart and great vessels in primary cardiac lymphoma. Eur J Echocardiogr 11: 74-76.

8. Su HY, Huang HL, Sun CM, Hou SM, Chen ML (2009) Primary cardiac lymphoma evaluated with integration of PET/CT and contrast-enhanced CT. Clin Nucl Med 34: 298-301. [Crossref]

Copyright: (C2017 Kara PO. This is an open-access article distributed under the terms of the Creative Commons Attribution License, which permits unrestricted use, distribution, and reproduction in any medium, provided the original author and source are credited. 